\title{
Perturbations and Stability of Rotating Stars. I. Completeness of Normal Modes
}

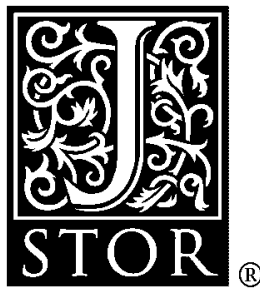

\author{
J. Dyson; B. F. Schutz
}

Proceedings of the Royal Society of London. Series A, Mathematical and Physical Sciences, Vol. 368, No. 1734. (Nov. 13, 1979), pp. 389-410.

Stable URL:

http://links.jstor.org/sici?sici=0080-4630\%2819791113\%29368\%3A1734\%3C389\%3APASORS\%3E2.0.CO\%3B2-X

Proceedings of the Royal Society of London. Series A, Mathematical and Physical Sciences is currently published by The Royal Society.

Your use of the JSTOR archive indicates your acceptance of JSTOR's Terms and Conditions of Use, available at http://www.jstor.org/about/terms.html. JSTOR's Terms and Conditions of Use provides, in part, that unless you have obtained prior permission, you may not download an entire issue of a journal or multiple copies of articles, and you may use content in the JSTOR archive only for your personal, non-commercial use.

Please contact the publisher regarding any further use of this work. Publisher contact information may be obtained at http://www.jstor.org/journals/rsl.html.

Each copy of any part of a JSTOR transmission must contain the same copyright notice that appears on the screen or printed page of such transmission.

The JSTOR Archive is a trusted digital repository providing for long-term preservation and access to leading academic journals and scholarly literature from around the world. The Archive is supported by libraries, scholarly societies, publishers, and foundations. It is an initiative of JSTOR, a not-for-profit organization with a mission to help the scholarly community take advantage of advances in technology. For more information regarding JSTOR, please contact support@jstor.org. 


\title{
Perturbations and stability of rotating stars.
}

\section{Completeness of normal modes}

\author{
By J. Dyson and B. F. Schutz \\ Department of Applied Mathematics and Astronomy, \\ University College, Cardiff, U.K.
}

\section{(Communicated by S. Chandrasekhar, F.R.S. - Received 19 March 1979)}

Linear adiabatic perturbations of a differentially rotating, axisymmetric, perfect-fluid stellar model have normal modes described by a quadratic eigenvalue problem of the form

$$
\left(\lambda^{2} A+\lambda B+C\right) \xi=0,
$$

where $A$ and $C$ are symmetric operators, $B$ antisymmetric, and $\xi$ the Lagrangian displacement vector. We study this problem and the associated time evolution equation. We show that, in the Hilbert space $H^{\prime}$, whose norm is square-integration weighted by $A$, the operators $A^{-1} B$ and $A^{-1} C$ are anti-selfadjoint and selfadjoint, respectively, when restricted to vectors $\xi$ belonging to a particular but arbitrary axial harmonic. We then find bounds on the spectrum of normal modes and show that any initial data in the domain of $C$ leads to a solution whose growth rate is limited by the spectrum and which can be expressed in a certain weak sense as a linear superposition of the normal modes. The normal modes are defined more precisely in terms of parallel projection operators associated with each isolated part of the spectrum. The quadratic eigenvalue problem can be reformulated in the space $H^{\prime} \oplus H^{\prime}$ (initial data space, or phase space) as a linear eigenvalue problem for an operator $T$, the generator of time evolution. This operator is not selfadjoint in $H^{\prime} \oplus H^{\prime}$ but it is selfadjoint in a Krein space (an indefinite inner-product space) formed by equipping $H^{\prime} \oplus H^{\prime}$ with the symplectic inner product. The normal modes are its eigenvectors and generalized eigenvectors.

\section{INTRODUCTION}

\section{(a) Motivation}

Interest in rotating stars and their perturbations has been growing rapidly during the past ten years. It is probably fair to say that the influence of angular momentum on dynamical phenomena is one of the most poorly understood areas of astrophysics, despite the fact that its importance on nearly every mass scale, from star formation to galactic structure, cannot be doubted. In attempting to gain some feeling for the effects of rotation, one of the simplest systems to study is the differentially rotating perfect-fluid star. An understanding of its principal instabilities should be relevant to the study of the collapse of massive stellar cores and the formation of pulsars. 
An understanding of its short-wavelength normal modes may be important for such objects as cataclysmic variables and $\beta$-Cephei (multi-period) variable stars (Papaloizou \& Pringle 1978). The large scale modes of rotation-dominated fluid disks have also been used to model spiral structure in galaxies (Bardeen I975). The perturbation problem itself has, moreover, considerable mathematical interest, being expressible either as a quadratic eigenvalue problem (equation (4.9) below) or as a linear eigenvalue problem for a non-selfadjoint operator (equation (4.10)), both of which are much less well understood than the linear selfadjoint eigenvalue problem that arises in non-rotating stars ( $\$ 2$ below).

The only fluid systems whose global perturbations have been at all well studied outside the slow rotation approximation are the Maclaurin spheroids (Chandrasekhar 1969) and the thin disk (Bardeen 1975). (For a local analysis see the review by Fricke \& Kippenhahn 1972.) This paper is the first of a series that will study, analytically and numerically, linear adiabatic perturbations of more general systems. We shall always take our stars to be axisymmetric perfect-fluid systems without meridional circulation. The series will focus attention on the normal modes of the stars, because at present they are the key to instability: there is no general criterion for non-axisymmetric dynamical instability. This situation is in contrast with perturbations of non-rotating stars (Lebovitz $1965 a, b$ ), with axisymmetric perturbations of rotating stars (Chandrasekhar \& Lebovitz I968), and with nonaxisymmetric secular instability of rotating stars (Bardeen et al. 1977), all of which have criteria based on the positive-definiteness of certain functionals over a given set of trial functions. Even the study of secular instability (that is, instability to viscosity and gravitational radiation), however, comes down to a study of normal modes because all perfect fluid models are formally unstable (Friedman \& Schutz r $978 a, b$ ). Only a detailed knowledge of the dynamical normal modes enables one to calculate whether the timescale of the secular instability is significant and whether the competing effects of viscosity and gravitational radiation cancel one another or not (Detweiler \& Lindblom I977; Comins 1978). But in order to have confidence in the results of a normal mode analysis one needs to know whether the modes are complete: can any perturbation be expressed as a superposition of normal modes? In this paper we show that the answer is yes.

\section{(b) The problem}

We consider unperturbed stars which are (i) perfect fluids with the density vanishing on the surface (the surface being defined by the pressure $p=0$ ), (ii) axisymmetric, (iii) in purely azimuthal flow described by an angular velocity $\Omega$. We allow $\Omega$ to take both signs inside the star and we do not assume reflexion symmetry through any plane perpendicular to the axis of rotation. Linear, adiabatic perturbations are governed by the following equations, written in terms of the Lagrangian displacement vector $\xi(x, t) \dagger$ in the reference frame of a non-rotating (inertial) observer

$\dagger$ For footnote see facing page. 
(Lynden-Bell \& Ostriker 1967):

$$
A \xi_{\mathrm{tt}}+B \xi_{\mathrm{t}}+C \xi=0
$$

where a subscript ' $t$ ' indicates a time derivative and where the operators $A, B$, and $C$ acting on an arbitrary vector $\boldsymbol{\eta}$ give

$$
\begin{aligned}
A \boldsymbol{\eta} & =\rho \boldsymbol{\eta}, \\
B \boldsymbol{\eta} & =2 \rho(\boldsymbol{v} \cdot \boldsymbol{\nabla}) \boldsymbol{\eta}, \\
C \boldsymbol{\eta} & =\rho(\boldsymbol{v} \cdot \boldsymbol{\nabla})^{2} \boldsymbol{\eta}-\boldsymbol{\nabla}(\gamma p \boldsymbol{\nabla} \cdot \boldsymbol{\eta}+\boldsymbol{\eta} \cdot \boldsymbol{\nabla} p)+(\boldsymbol{\nabla} p)(\boldsymbol{\nabla} \cdot \boldsymbol{\eta}) \\
& \quad+(\boldsymbol{\eta} \cdot \boldsymbol{\nabla})(\boldsymbol{\nabla} p)+\rho(\boldsymbol{\eta} \cdot \boldsymbol{\nabla}) \boldsymbol{\nabla} \Phi+\rho \boldsymbol{\nabla} \delta_{\eta} \Phi .
\end{aligned}
$$

Here $\gamma$ is the adiabatic index $(\partial \ln p / \partial \ln \rho)_{S}$ and $\delta_{\eta} \Phi$ is the solution to

$$
\nabla^{2} \delta_{\eta} \Phi=4 \pi G \delta_{\eta} \rho=-4 \pi G \boldsymbol{\nabla} \cdot(\rho \boldsymbol{\eta}) .
$$

We assume that the density $\rho$, pressure $p$, velocity $\boldsymbol{v}$, and gravitational field $\Phi$ of the unperturbed model are as smooth as necessary (infinitely differentiable will always do) and satisfy the equation of hydrostationary equilibrium

and Poisson's equation

$$
\rho(\boldsymbol{v} \cdot \boldsymbol{\nabla}) \boldsymbol{v}+\nabla p+\rho \boldsymbol{\nabla} \Phi=0,
$$

$$
\nabla^{2} \Phi=4 \pi G \rho .
$$

We make no assumption about the equation of state. Equation (1.1) has 'physical' solutions and a set of 'trivial' solutions which arise from a certain ambiguity in the relation between initial data for $\xi$ and for the Eulerian perturbations (Friedman \& Schutz $1978 a$ ). Our completeness result applies to the whole set of solutions. Moreover, it would be unaffected if (1.2) were expressed with respect to some rigidly rotating reference frame.

A normal mode is usually understood to be a solution to (1.1) with time dependence $\mathrm{e}^{\lambda t}$ :

$$
\begin{gathered}
\xi(x, t)=\xi(x) \mathrm{e}^{\lambda t}, \\
\left(\lambda^{2} A+\lambda B+C\right) \xi(x)=0 .
\end{gathered}
$$

We shall follow the rather unusual convention of using simply $\lambda$ in the exponential, rather than $i \omega$ or $-i \omega$. This will be convenient for our Laplace-transform approach to the initial-value problem in $\S 4$. Note that, since $\lambda$ is generally complex, $\xi(x)$ is also complex, and the solution is the real part of the product $\xi(x) \exp \lambda t$. Our definition of a normal mode must, however, be refined further to allow for the continuous spectrum, and this is done in $\S 5$.

Because the star is axisymmetric, perturbations can be expanded in 'axial

$\dagger$ The equations in their Eulerian form are convenient for some purposes, particularly since they have only first order spatial derivatives. However, the Lagrangian version is superior for the present study because of the symmetry of its differential operators (equations (1.12)(1.14)). 
harmonics' of eigenvalue $m$. In a cylindrical or spherical coordinate system $\boldsymbol{\xi}(\boldsymbol{x})$ has components

$$
\xi^{k}\left(x_{1}, x_{2}, \phi\right)=\xi_{m}^{k}\left(x_{1}, x_{2}\right) \mathrm{e}^{\mathrm{i} m \phi}
$$

where $\phi$ is the angle about the axis of symmetry (always the $z$-axis when we label it explicitly). Equivalently, in the coordinate-free language of differential geometry we require

$$
\mathscr{L}_{\phi} \xi=\mathrm{i} m \xi,
$$

where $\mathscr{L}_{\phi}$ is the Lie derivative with respect to the generator of rotations about the axis of symmetry. Since the axial harmonics are complete, we lose no generality looking at fixed $m$. Restricted to such vector fields, the operator $A$ is unchanged but $B$ becomes simpler because of the fact that $v$ is purely azimuthal. The restriction of $B$ can be written

$$
B_{m} \xi_{m}=2 \rho \Omega\left(\mathrm{i} m \xi_{m}+e_{z} \times \xi_{m}\right)
$$

where $\Omega$ is the star's angular velocity and $e_{z}$ is the unit vector along the axis of symmetry. In the same way, $C$ becomes $C_{m}$, whose rather complicated form we do not need to write out explicitly. From now on we shall always assume a fixed $m$ and write $\xi_{m}\left(x_{1}, x_{2}\right), B_{m}$, and $C_{m}$ simply as $\xi, B$, and $C$.

\section{(c) Functional analysis in this problem}

Completeness is a question for functional analysis, and inevitably much of this paper is rather technical. In this section we define some terms and conventions we follow, and try to explain the basic ideas to readers who want to understand our result but are unfamiliar with the mathematics. We follow the well-written Riesz \& Sz.-Nagy (1955) wherever possible.

The usual inner product between vector fields $\boldsymbol{\xi}$ and $\boldsymbol{\eta}$,

$$
(\xi, \boldsymbol{\eta})=\int \xi^{*} \cdot \boldsymbol{\eta} \mathrm{d} V
$$

(where * means complex conjugation and the integral is over the volume of the star) and its associated norm

$$
\|\xi\|=(\xi, \xi)^{\frac{1}{2}}
$$

define the Hilbert space $H$ of vector fields $\xi$ of finite norm (1.10). An operator $D$ in $H$ whose domain is dense in $H$ (which means that any vector $\boldsymbol{\eta}$ in $H$ can be approximated by a vector $\boldsymbol{\eta}_{1}$ in the domain of $D$ in such a way that $\left\|\boldsymbol{\eta}-\boldsymbol{\eta}_{1}\right\|$ is as small as desired) has an adjoint $D^{*}$ whose action on a vector $\eta, D^{*} \eta$, is defined by

$$
\left(D^{*} \boldsymbol{\eta}, \boldsymbol{\xi}\right) \equiv(\boldsymbol{\eta}, D \xi) \forall \boldsymbol{\xi} \epsilon \quad \text { domain of } D .
$$

The domain of $D^{*}$ is the set of vectors $\boldsymbol{\eta}$ in $H$ for which $(\boldsymbol{\eta}, D \xi)$ is finite for all $\boldsymbol{\xi}$ in the domain of $D$. If $D$ is a differential operator then the domain of $D^{*}$ may clearly be considerably larger than that of $D$. 
Lynden-Bell \& Ostriker (1967) show that $A, B$, and $C$ have the following properties on vector fields $\boldsymbol{\xi}$ and $\boldsymbol{\eta}$ both in the domain of the relevant operator:

$$
\begin{aligned}
& (\boldsymbol{\eta}, A \xi)=(A \boldsymbol{\eta}, \boldsymbol{\xi}), \\
& (\boldsymbol{\eta}, B \xi)=-(B \boldsymbol{\eta}, \boldsymbol{\xi}), \\
& (\boldsymbol{\eta}, C \boldsymbol{\xi})=(C \boldsymbol{\eta}, \boldsymbol{\xi}) .
\end{aligned}
$$

Then $A$ and $C$ are said to be symmetric and $B$ antisymmetric. They are not necessarily selfadjoint (identical to their adjoints) because their domains may be smaller than their adjoints' domains. In fact equations $(1.2 a)$ and (1.8) show that the domains of $A$ and $B$ (and of their adjoints) are all of $H$, so they are, respectively, selfadjoint and anti-selfadjoint. This is not so for $C$, which will be studied in $\S 3$.

Because $A$ is positive we can define another inner product, which we denote by angle brackets

$$
\langle\boldsymbol{\xi}, \boldsymbol{\eta}\rangle=\int \boldsymbol{\xi}^{*} \cdot A \boldsymbol{\eta} \mathrm{d} V=(\boldsymbol{\xi}, A \boldsymbol{\eta})
$$

The inner product $\langle$,$\rangle defines a Hilbert space H^{\prime}$ which is larger than $H$ because $\rho$ vanishes on the surface: there are some functions which have a finite integral weighted by $\rho$ (i.e. in $H^{\prime}$ ) but an infinite unweighted integral (i.e. not in $H$ ). We shall use the density-weighted inner product (1.15) and its norm extensively.

Finally, the norm of an operator $D$ is defined in terms of whatever norm \|\| is defined for vectors:

$$
\|D\|=\sup [\|D \xi\| /\|\xi\|],
$$

where 'sup' means the least upper bound for all $\xi$ in the relevant Hilbert space. The operators $A$ and $B$ are said to be bounded because they have finite norms.

\section{(d) Outline of the paper}

The first step in our completeness proof is the proof in $\S 3$ that $C$ is selfadjoint in $H^{\prime}$. This is followed in $\S 4$ by a study of the initial-value problem. We put bounds on the growth rate of any perturbation and bounds on the spectrum of eigenfrequencies. In $\S 5$ we define the normal modes precisely and show that any perturbation can be resolved as a superposition of contributions from each isolated part of the spectrum. In order to motivate our approach and also to show the limitations of what we have been able to prove, we first take a look at the completely solved problem of the normal modes of a non-rotating star.

\section{NORMAL MODES OF A SPHERICAL, NON-ROTATING STAR}

We specialize equations (1.1) and (1.5) to the spherical background star by setting the operator $B$ to zero:

$$
\begin{gathered}
A \xi_{\mathrm{tt}}+C \xi=0, \\
\lambda^{2} A \xi+C \xi=0 .
\end{gathered}
$$


An important point is that (2.2) is not solved by the eigenvectors and eigenvalues of $C$, but rather by those of $A^{-1} C$ :

$$
-\lambda^{2} \xi=A^{-1} C \xi \equiv C^{\prime} \xi,
$$

where we have introduced the shorthand $C^{\prime}$ for $A^{-1} C$. But $C^{\prime}$ is not a symmetric operator in the usual inner product (, ), and this is the reason we introduced the density-weighted inner product $\langle$,$\rangle and its Hilbert space H^{\prime}$. It is easy to see that symmetry of $C^{\prime}$ in the inner product $\langle$,$\rangle follows from symmetry of C$ and $A$ in (, ):

$$
\begin{aligned}
\left\langle\eta, C^{\prime} \xi\right\rangle & =\left\langle\eta, A^{-1} C \xi\right\rangle=\left(\eta, A A^{-1} C \xi\right)=(\eta, C \xi)=(C \eta, \xi) \\
& =\left(A A^{-1} C \eta, \xi\right)=\left(A^{-1} C \eta, A \xi\right)=\left(C^{\prime} \eta, A \xi\right)=\left\langle C^{\prime} \eta, \xi\right\rangle .
\end{aligned}
$$

So the problem reduces to studying the eigenvectors and eigenvalues of the symmetric operator $C^{\prime}$ in $H^{\prime}$.

The question of the completeness of these eigenvectors turns on whether $C^{\prime}$ is not merely symmetric but selfadjoint (cf. Riesz \& Sz.-Nagy 1955). There are several possible approaches to this problem. Eisenfeld (1969) has proved it for non-rotating stars more or less directly. It also is a special case of our general proof for rotating stars in $\S 3$ below. What selfadjointness guarantees is the existence of a family of orthogonal projection operators $\left\{E_{\mu}\right\}$, depending on the real number $\mu$. One can think of $E_{\mu}$ as projecting onto the subspace of $H^{\prime}$ spanned by all the eigenvectors associated with eigenvalues less than or equal to $\mu$. Similarly, the operator $\mathrm{d} E_{\mu}$ is the difference between $E_{\mu}$ and $E_{\mu+\mathrm{d} \mu}$, and so projects onto eigenvectors whose eigenvalues lie between $\mu$ and $\mu+\mathrm{d} \mu$. If the spectrum is discrete, which is usually the case for spherical stars, then if $\mu_{0}$ is an eigenvalue $\mathrm{d} E_{\mu_{0}}$ projects onto a subspace of the domain of $C^{\prime}$. But if the spectrum has a continuous portion (which does not seem to be excluded), then the 'eigenvectors' need not be in the domain of $C^{\prime}$. The projections $\mathrm{d} E_{\mu}$ will, nevertheless, be complete, in the sense that their sum is unity:

$$
\int_{-\infty}^{\infty} \mathrm{d} E_{\mu}=I \text {. }
$$

A relation like this will also be derived for rotating stars. However, for nonrotating stars we can make yet another step. Because $C^{\prime}$, when operating on vectors in the eigensubspace defined by $\mathrm{d} E_{\mu}$, just has the value $\mu$, we have a spectral theorem:

$$
\begin{gathered}
C^{\prime}=\int_{-\infty}^{\infty} \mu \mathrm{d} E_{\mu}, \\
\left(C^{\prime}\right)^{2}=\int_{-\infty}^{\infty} \mu^{2} \mathrm{~d} E_{\mu},
\end{gathered}
$$

and so on for polynomials and regular functions of $C^{\prime}$.

How does this relate to solutions of (2.1)? If the solution is analytic in time at $t=0$ then we have the Taylor series

$$
\xi(t)=\xi(0)+t \xi_{\mathrm{t}}(0)+\frac{1}{2 !} t^{2} \xi_{\mathrm{tt}}(0)+\ldots,
$$


all the coefficients of which are determined by $\xi(0)$ and $\xi_{t}(0)$ by the equations

etc. This gives

$$
\begin{aligned}
\xi_{\mathrm{tt}}(0) & =-C^{\prime} \xi(0), \\
\xi_{\mathrm{ttt}}(0) & =-C^{\prime} \xi_{\mathrm{t}}(0),
\end{aligned}
$$

$$
\xi(t)=\left[I-\frac{1}{2 !} t^{2} C^{\prime}+\frac{1}{4 !} t^{4}\left(C^{\prime}\right)^{2}-+\ldots\right] \xi(0)+\left[t I-\frac{1}{3 !}{ }^{3} t C^{\prime}+\frac{1}{5 !} t^{5}\left(C^{\prime}\right)^{2}-+\ldots\right] \xi_{\mathrm{t}}(0) .
$$

By the spectral theorem (2.6), we can write this as

$$
\begin{aligned}
\xi(t)= & {\left[\int_{-\infty}^{\infty}\left(1-\frac{1}{2 !} t^{2} \mu+\frac{1}{4 !} t^{4} \mu^{2}-+\ldots\right) \mathrm{d} E_{\mu}\right] \xi(0) } \\
& +\left[\int_{-\infty}^{\infty}\left(t-\frac{1}{3 !} t^{3} \mu+\frac{1}{5 !} t^{5} \mu^{2}-+\ldots\right) \mathrm{d} E_{\mu}\right] \xi_{\mathrm{t}}(0) \\
= & \frac{1}{2}\left[\int_{-\infty}^{\infty}\left(\mathrm{e}^{\mathrm{i} \mu^{\frac{1}{2}} t}+\mathrm{e}^{-\mathrm{i} \mu^{\mathrm{l}} t}\right) \mathrm{d} E_{\mu}\right] \xi(0)+\frac{1}{2 \mathrm{i}}\left[\int_{-\infty}^{\infty} \mu^{-\frac{1}{2}}\left(\mathrm{e}^{\mathrm{i} \mu^{\frac{1}{2}} t}-\mathrm{e}^{-\mathrm{i} \mu^{\frac{1}{t}} t}\right) \mathrm{d} E_{\mu}\right] \xi_{\mathrm{t}}(0) .
\end{aligned}
$$

The second form is really just a shorthand for the first, but it can be rearranged in an illustrative form:

$$
\xi(t)=\frac{1}{2}\left[\int_{-\infty}^{\infty} e^{\mathrm{i} \mu^{\frac{1}{t}} t} \mathrm{~d} E_{\mu}\left\{\xi(0)+\frac{1}{\mathrm{i} \mu^{\frac{1}{2}}} \xi_{\mathrm{t}}(0)\right\}\right]+\frac{1}{2}\left[\int_{-\infty}^{\infty} \mathrm{e}^{-\mathrm{i} \mu^{\mathrm{t}} t} \mathrm{~d} E_{\mu}\left\{\xi(0)-\frac{1}{\mathrm{i} \mu^{\frac{1}{2}}} \xi_{\mathrm{t}}(0)\right\}\right] .
$$

This shows explicitly how a spectral value $\mu$ defines two eigenfrequencies $\pm \mu^{\frac{1}{2}}$, which are related to solutions having time dependence $\mathrm{e}^{ \pm \mathrm{i} \mu \frac{1}{2} t}$ and which evolve from specific initial data. Thus, if initial data are chosen for which $\xi_{t}(0)=i \mu^{\frac{1}{2}} \xi(0)$ for some $\mu$ in the spectrum, then the time dependence $e^{-\mathrm{i} \mu \frac{1}{2} t}$ will be absent. So equation (2.10) is the representation of $\xi(t)$ in terms of normal modes: the integral over $\mu$ is the linear superposition of modes, and each mode is defined by a particular choice of data for $\xi(0)$ and $\xi_{\mathrm{t}}(0)$. The distinction between normal modes, which involve both $\xi(0)$ and $\xi_{\mathrm{t}}(0)$, and eigenfunctions of $C^{\prime}$ is often blurred, but it will be crucial to an understanding of the normal modes of rotating stars.

We show in $\S 3$ that, under reasonable assumptions, $C^{\prime}$ has a lower bound. From (2.10) this sets an upper bound on the growth rate of any perturbation. Note also that (2.9) and (2.10) are apparently not well defined if $\mu=0$ is in the spectrum. This case is handled by referring directly to (2.8), which shows that such a 'zerofrequency mode' is a linear function of time.

\section{SELFADJOINTNESS OF OPERATORS OF A ROTATING STAR}

As in the non-rotating case we define operators

$$
B^{\prime}=A^{-1} B \quad \text { and } \quad C^{\prime}=A^{-1} C,
$$

and write the eigenvalue problem as

$$
\lambda^{2} \xi+\lambda B^{\prime} \xi+C^{\prime} \xi=0,
$$


where the operators have the symmetry properties

$$
\begin{aligned}
& \left\langle\eta, B^{\prime} \xi\right\rangle=-\left\langle B^{\prime} \eta, \xi\right\rangle, \\
& \left\langle\eta, C^{\prime} \xi\right\rangle=\left\langle C^{\prime} \eta, \xi\right\rangle,
\end{aligned}
$$

in $H^{\prime}$, when both $\eta$ and $\xi$ are in the appropriate domains.

It is easy to show that $B^{\prime}$ is bounded, i.e. that $\dagger^{\prime}$

$$
b(\xi) \equiv\left\langle\xi, B^{\prime} \xi\right\rangle /\langle\xi, \xi\rangle
$$

is a bounded function of $\xi$ on all of $H^{\prime}$. By (1.8) this is

$$
b(\xi)=2 \int \rho \Omega \xi^{*} \cdot\left(\operatorname{Im} \xi+\boldsymbol{e}_{z} \times \boldsymbol{\xi}\right) \mathrm{d} V / \int \rho \boldsymbol{\xi}^{*} \cdot \boldsymbol{\xi} \mathrm{d} V,
$$

the integrals being taken over the whole star. Since

$$
\left|\xi^{*} \cdot\left(e_{z} \times \xi\right)\right|=\left|\left(\xi^{*} \times \xi\right) \cdot e_{z}\right| \leqslant \xi^{*} \cdot \xi,
$$

it follows that

$$
|b(\xi)| \leqslant 2(m+1) \Omega_{\max }
$$

where $\Omega_{\max }$ is the maximum value of $|\Omega|$ in the star. So $B^{\prime}$ is a bounded antisymmetric operator whose domain is $H^{\prime}$; it is therefore anti-selfadjoint.

The operator $C^{\prime}$ is not bounded, so we must prove it is selfadjoint (more properly, that it has an extension to a larger domain such that the domains of it and its adjoint are identical). We use the Stone-Friedrichs theorem (cf. Riesz \& Sz.-Nagy 1955): if $C^{\prime}$ is symmetric on a dense domain and if the real function

$$
c(\xi)=\left\langle\xi, C^{\prime} \xi\right\rangle /\langle\xi, \xi\rangle
$$

has a lower (upper) bound on the domain of $C^{\prime}$, then there exists a selfadjoint extension of $C^{\prime}$ with the same lower (upper) bound. (After proving the existence of this extension, we will always take the symbol $C^{\prime}$ to refer to the extended operator.)

It is clear that $C^{\prime}$ has no upper bound, for consider the single term

$$
-\int \xi^{*} \cdot \nabla(\gamma p \nabla \cdot \xi) \mathrm{d} V=\int \gamma p|\nabla \cdot \xi|^{2} \mathrm{~d} V
$$

in which we have integrated by parts and used the fact that $p=0$ on the surface. We can certainly choose $\boldsymbol{\xi}$ to have unit norm but very short-wavelength oscillations across the star; the shorter the wavelength the larger the integral. As this is the only term in $C^{\prime}$ containing second derivatives, it will not be compensated by other terms. (Physically, one should expect this unboundedness, for sound waves must have the local dispersion relation (frequency) $=($ sound speed $) /($ wavelength $)$, which associates large frequencies with short wavelengths.)

We shall show that $C^{\prime}$ has a lower bound. Hunter (I977) showed that $C$ has a lower bound in $H$, but that does not suffice for us, because our space $H^{\prime}$ is larger

$\dagger$ This definition of boundedness is equivalent to that using (1.16) for symmetric and antisymmetric operators. 
than $H$. Specifically, our denominator in $c(\xi)$ contains $\rho$, which vanishes on the surface, and it is conceivable that by concentrating $\xi$ there one could make $-c(\xi)$ arbitrarily large. In fact this $i s$ possible without a further (weak) restriction on $C$.

The restriction is the following condition on the unperturbed model: the square of the generalized Brunt-Viäsäla frequency (local buoyancy frequency) associated with a real displacement $\xi$,

$$
N^{2}(\xi)=(\xi \cdot \nabla p)(\xi \cdot \mathscr{S}) / \rho|\xi|^{2},
$$

must be bounded below for all $\xi$ uniformly over the star. Here $\mathscr{S}$ is the vector Schwarzschild discriminant:

$$
\mathscr{S}=\frac{1}{\rho} \nabla \rho-\frac{1}{\gamma p} \nabla p=\left(\frac{\partial \ln \rho}{\partial S}\right)_{p} \nabla S
$$

In appendix A we show that $N^{2}$ is the square of the frequency characterizing the response of a local perturbation that preserves the local angular velocity. If $N^{2}$ becomes arbitrarily large and negative as, say, one approaches the surface of the star, then the star will have local instabilities of a very rapid nature and the model cannot be taken seriously. We believe that any reasonable model will satisfy this criterion. (We want to emphasize that our condition is only that $N^{2}$ have a lower bound, not that it be positive-definite or have any other particular value as its bound.)

To show how this condition guarantees lower-boundedness of $C^{\prime}$ we rewrite equation $(1.2 c)$ in the following form:

$$
C \boldsymbol{\xi}=\rho(\boldsymbol{v} \cdot \boldsymbol{\nabla}) \boldsymbol{\xi}+\rho \boldsymbol{\nabla}\left(\delta_{\xi} \Phi\right)-\rho(\boldsymbol{\xi} \cdot \boldsymbol{\nabla})(\boldsymbol{v} \cdot \boldsymbol{\nabla}) \boldsymbol{v}+\rho \delta_{\xi}\left(\frac{1}{\rho} \boldsymbol{\nabla} p\right),
$$

where $\delta_{\xi}$ denotes the Eulerian perturbation caused by the Lagrangian displacement $\xi$. Denoting the four terms in (3.9) by $C_{1} \xi, C_{2} \xi$, etc. in sequence, we also define

$$
C_{j}^{\prime}=A^{-1} C_{j}, \quad c_{j}(\xi)=\left\langle\xi, C_{j}^{\prime} \xi\right\rangle /\langle\xi, \xi\rangle, \quad j=1, \ldots, 4 .
$$

From now on we take $\xi$ to be real: a symmetric operator bounded on real vectors in its domain has the same bound on complex vectors.

We want to show that each $c_{j}$ is bounded below. The first three follow from arguments given by Hunter (1977; appendix A). The results are

$$
\begin{aligned}
& \text { (i) } c_{1}(\xi)\langle\xi, \xi\rangle=\left(\xi, \rho(\boldsymbol{v} \cdot \nabla)^{2} \xi\right) \geqslant-(m+1)^{2} \Omega_{\max }^{2}\langle\xi, \xi\rangle, \\
& \text { (ii) } c_{2}(\xi)\langle\xi, \xi\rangle=\left(\xi, \rho \nabla\left(\delta_{\xi} \Phi\right)\right) \geqslant-4 \pi G \rho_{\max }\langle\xi, \xi\rangle, \\
& \text { (iii) } c_{3}(\xi)\langle\xi, \xi\rangle=(\xi,-\rho(\xi \cdot \nabla)(\boldsymbol{v} \cdot \nabla) v) \geqslant \beta\langle\xi, \xi\rangle .
\end{aligned}
$$

Here $\Omega_{\max }$ and $\rho_{\max }$ are maximum angular velocity and density in the star; the constant $\beta$ in (3.13) depends on bounds on the derivatives of $\Omega$, and is evaluated in appendix B. 
For $c_{4}(\xi)$ we follow Hunter up to a point. The numerator of $c_{4}(\xi)$ is

$$
\begin{aligned}
\left(\xi, \rho \delta_{\xi}\left(\frac{1}{\rho} \nabla p\right)\right)= & \int\left[-\rho^{-1} \delta_{\xi} \rho(\xi \cdot \nabla p)+\xi \cdot \nabla\left(\delta_{\xi} p\right)\right] \mathrm{d} V \\
= & \int\left[\rho^{-1} \boldsymbol{\nabla} \cdot(\rho \xi)(\xi \cdot \nabla p)+(\xi \cdot \nabla)(-\gamma p \nabla \cdot \xi-\xi \cdot \nabla p)\right] \mathrm{d} V \\
= & \int\left[(\boldsymbol{\nabla} \cdot \boldsymbol{\xi})(\xi \cdot \nabla p)+\rho^{-1}(\xi \cdot \nabla p)(\xi \cdot \nabla \rho)\right. \\
& \quad-\boldsymbol{\nabla} \cdot(\gamma p \xi(\nabla \cdot \xi)+\xi(\xi \cdot \nabla p))+(\gamma p \nabla \cdot \xi+\xi \cdot \nabla p)(\nabla \cdot \xi)] \mathrm{d} V .
\end{aligned}
$$

The third term in the final integral is a pure divergence which vanishes upon integration because both $p$ and $\boldsymbol{\nabla} p$ vanish at the surface. So we may write

$$
\begin{aligned}
\left(\xi, \rho \delta_{\xi}\left(\frac{1}{\rho} \nabla p\right)\right) & =\int\left[\gamma p(\nabla \cdot \xi)^{2}+2(\xi \cdot \nabla p)(\nabla \cdot \xi)+\rho^{-1}(\xi \cdot \nabla \rho)(\xi \cdot \nabla p)\right] \mathrm{d} V \\
= & \int\left[\left|(\gamma p)^{\frac{1}{2}} \nabla \cdot \xi+\frac{\xi \cdot \nabla p}{(\gamma p)^{\frac{1}{2}}}\right|^{2}+\frac{\xi \cdot \nabla p}{\rho}\left(\xi \cdot \nabla \rho-\frac{\rho}{\gamma p} \xi \cdot \nabla p\right)\right] \mathrm{d} V \\
& \geqslant \int \rho^{-1}(\xi \cdot \nabla p)(\xi \cdot \mathscr{S}) \rho \mathrm{d} V=\int N^{2} \rho|\xi|^{2} \mathrm{~d} V .
\end{aligned}
$$

This means that

$$
c_{4}(\xi) \geqslant \int N^{2}(\xi) \rho|\xi|^{2} \mathrm{~d} V / \int \rho|\xi|^{2} \mathrm{~d} V \geqslant N_{\min }^{2},
$$

which brings in the assumed lower bound on $N^{2}$.

Summarizing, we have proved that

$$
\left\langle\xi, C^{\prime} \xi\right\rangle /\langle\xi, \xi\rangle \geqslant-(m+1)^{2} \Omega_{\max }^{2}-4 \pi G \rho_{\max }+N_{\min }^{2}+\beta .
$$

The only term that involves $m$ is, in fact, the first. Clearly, this lower bound is not uniform in $m$, a fact that has been noticed by several workers (Stewart 1975; Friedman \& Schutz 1978b). Only by restricting attention to a particular value of $m$ can we make this proof of the selfadjointness of $C^{\prime}$ work. But we want to make it clear that $C^{\prime}$ may still be selfadjoint on the larger Hilbert space which allows general azimuthal behaviour (or on $H^{\prime}$ even if $N^{2}(\xi)$ is not bounded below); only our method of proving it fails. It may be of some interest to note here also that (if $N^{2}(\xi)$ is bounded below) the operator $C^{\prime}-\frac{1}{4}\left(B^{\prime}\right)^{2}$ is bounded below uniformly in $m$ (i.e. regardless of azimuthal dependence) because it is simply $C^{\prime}$ without the term we called $C_{1}^{\prime}$ above. It is tempting to hope that this limits the growth rate of unstable modes, but we know of no argument which establishes this when $B^{\prime}$ and $C^{\prime}$ do not commute. 


\section{The EVOLUTION OF a GeNERAL PERTURBATION}

(a) General remarks

In this section we take the major steps toward the completeness result by finding an explicit expression for $\xi(t)$ in terms of $\xi(0)$ and $\xi_{t}(0)$. Our approach is to use Laplace transforms. In order to establish our results rigorously, we need to prove the existence of the inverse Laplace transform, and for this reason this section will be more technical than the previous two.

Our object is to study solutions to the equation

$$
\xi_{\mathrm{tt}}+B^{\prime} \xi_{\mathrm{t}}+C^{\prime} \boldsymbol{\xi}=0
$$

where $B^{\prime}$ and $C^{\prime}$ have the following properties in a Hilbert space $H^{\prime}$ with inner product $\langle$,$\rangle :$

(i) $B^{\prime}$ is anti-selfadjoint and bounded;

(ii) $C^{\prime}$ is selfadjoint with lower bound $s>-\infty$.

This problem has been studied extensively in the literature, and results more powerful than those developed here have been obtained under more restrictive assumptions: $H^{\prime}$ finite-dimensional or $C^{\prime}$ positive-definite, for example (cf. Barston s967-1974; Bognar 1974). We believe our results are the strongest so far to be applicable to the stellar perturbation problem.

It is sometimes convenient to rewrite (4.1) as the two first order equations

$$
\frac{\mathrm{d}}{\mathrm{d} t}\left(\begin{array}{l}
\xi \\
\eta
\end{array}\right)=\left(\begin{array}{cc}
0 & 1 \\
-C^{\prime} & -B^{\prime}
\end{array}\right)\left(\begin{array}{l}
\xi \\
\eta
\end{array}\right)
$$

which can be written compactly as

$$
\zeta_{t}=T \zeta
$$

Here $T$ is the matrix in (4.4) and $\zeta$ is an element of the Hilbert space $H_{2}=H^{\prime} \oplus H^{\prime}$ with inner product $\langle\langle\rangle$,

$$
\left\langle\left\langle\zeta_{1}, \zeta_{2}\right\rangle\right\rangle \equiv\left\langle\xi_{1}, \xi_{2}\right\rangle+\left\langle\boldsymbol{\eta}_{1}, \boldsymbol{\eta}_{2}\right\rangle
$$

where $\boldsymbol{\xi}_{i}$ and $\boldsymbol{\eta}_{i}$ are the components of $\zeta_{i}$. The space $H_{2}$ may be regarded as initialdata space. The inner product (4.6), though mathematically acceptable, is clearly not physically meaningful: if by (4.4) we regard $\boldsymbol{\eta}$ as the time derivative of $\xi$, then it is not even dimensionally correct. A more meaningful inner product is given by the matrix

$$
W=\left(\begin{array}{cr}
-B^{\prime} & -1 \\
1 & 0
\end{array}\right)
$$

which is the symplectic structure of the problem. With the indefinite inner product $W, H_{2}$ becomes phase space. With respect to $W$, the matrix $T$ is selfadjoint; equivalently, the matrix $E=W T$,

$$
E=\left(\begin{array}{cc}
C^{\prime} & 0 \\
0 & 1
\end{array}\right)
$$


is selfadjoint with respect to $\langle\langle\rangle$,$\rangle . Obviously E$ is just the conserved energy of the perturbation (its canonical energy, as discussed at length by Friedman \& Schutz I $978 a, b)$. However, because $W$ is not positive-definite, it is not possible to define a Hilbert space by using this natural inner product as a norm. Because it is nondegenerate, $W$ defines a Krein space (Bognar 1974), which is an indefinite inner product space. The operator $T$ is selfadjoint in the Krein space. Conversely, it should not be surprising that $T$ is not symmetric with respect to $\langle\langle\rangle$,$\rangle , which has$ no natural role in the problem. Nevertheless, we will use $\langle\langle\rangle$,$\rangle exclusively from$ now on. The symmetry of $T$ is of less interest to us than the completeness of its eigenvectors, and to discuss eigenvectors and completeness one needs only the topology of $H_{2}$, not its metric structure. The inner product $\langle\langle\rangle$,$\rangle induces at least$ as fine a topology as one can define using $W$ (cf. Bognar 1974).

\section{(b) The eigenvector problem}

There are two equivalent eigenvalue problems,

$$
\left(\lambda^{2}+\lambda B^{\prime}+C^{\prime}\right) \xi=0
$$

and

$$
(\lambda-T) \zeta=0 .
$$

We shall prove their equivalence below. First we need some definitions. The set of operators

$$
L(\lambda)=\lambda^{2}+\lambda B^{\prime}+C^{\prime}
$$

is called a quadratic pencil (Bognar 1974). Its resolvent set $\rho(L)$ is the set of complex numbers $\lambda$ for which $[L(\lambda)]^{-1}$ exists as a bounded operator with domain $H^{\prime}$. When $\lambda$ is in $\rho(L)$, the operator-valued function of $\lambda$

$$
R_{\lambda}(L)=[L(\lambda)]^{-1}
$$

is the resolvent of the pencil. The complement of $\rho(L)$ in the complex plane is the spectrum of the pencil, $\sigma(L)$. All these are analogues of the definitions for the linear eigenvalue problem (4.10) in $H_{2}$ with inner product (4.6). The resolvent set of $T$, $\rho(T)$, is the set of complex $\lambda$ for which the resolvent of $T$,

$$
R_{\lambda}(T) \equiv(\lambda-T)^{-1},
$$

is a bounded operator with domain $H_{2}$. The spectrum of $T, \sigma(T)$, is the complement of $\rho(T)$.

We now show that the eigenvalue problems are equivalent, in the sense that

The formal inverse $R_{\lambda}(T)$ is

$$
\sigma(T)=\sigma(L) .
$$

$$
R_{\lambda}(T)=R_{\lambda}(L)\left(\begin{array}{cc}
\lambda+B & 1 \\
-C^{\prime} & \lambda
\end{array}\right) ;
$$

simple multiplication shows that it is indeed the left inverse of $\lambda-T$ on the domain of $T$ if $\lambda \in \rho(L)$. If the coefficients in $C^{\prime}$ and $B^{\prime}$ are $C^{\infty}$ functions on the star, they will map the space of $C^{\infty}$ vectors into itself; on this subspace of the domain of $T$, equation 
(4.15) defines the true inverse of $\lambda-T$ if $\lambda \in \rho(L)$. But the r.h.s. is a bounded operator if $\lambda \in \rho(L)$ : the only possibly unbounded term is $R_{\lambda}(L) C^{\prime}$, which equals

$$
R_{\lambda}(L) C^{\prime}=1-R_{\lambda}(L)\left(\lambda^{2}+\lambda B^{\prime}\right)
$$

on the domain of $C^{\prime}$. The r.h.s. is bounded because $R(L)$ is bounded, which enables one to extend $R_{\lambda}(L) C^{\prime}$ as a bounded operator to the whole of $H^{\prime}$. This proves that if $\lambda \in \rho(L), R_{\lambda}(T)$ is the bounded inverse of $(\lambda-T)$ and so $\lambda \in \rho(T)$. On the other hand, it is well known (Riesz \& Sz.-Nagy 1955) that $R_{\lambda}(T)$ is an analytic operator-valued function on $\rho(T)$, and it is easy to construct a similar proof that $R_{\lambda}(L)$ is analytic on $\rho(L)$, so that the r.h.s. of (4.15) is analytic on $\rho(L)$. Therefore the two resolvent sets must in fact be identical, which proves (4.14).

The proof that $R_{\lambda}(L)$ is analytic yields another result that we shall need below: if $\mathrm{d}(\lambda)$ is the distance of $\lambda \in \rho(L)$ from the nearest point in $\sigma(L)$, then

$$
\left\|R_{\lambda}(L)\right\| \geqslant 1 / \mathrm{d}(\lambda)\left[\mathrm{d}(\lambda)+\left\|2 \lambda+B^{\prime}\right\|\right]
$$

where the norm \|\| is defined by the inner product $\langle$,$\rangle .$

In solving the initial-value problem we shall concentrate on $L(\lambda)$, returning to consider $T$ in $\S 5$ on normal modes. For the rest of $\S 4$ we write $R_{\lambda}(L)$ simply as $R(\lambda)$.

It is possible to show that both $\sigma(L)$ and $\rho(L)$ are non-empty. In particular, points $\lambda$ on the positive real axis satisfying the inequality

$$
\lambda^{2}+\lambda\left\|B^{\prime}\right\|+s>0
$$

(recall that $s$ is the lower bound of $C^{\prime}$ ) are in the resolvent. We prove this by defining $H=\lambda^{2}+C^{\prime}$, which is positive-definite by (4.18) and is selfadjoint, and expanding

$$
\begin{aligned}
R(\lambda)=[L(\lambda)]^{-1}=\left[H+\lambda B^{\prime}\right]^{-1}=H^{-\frac{1}{2}} & {\left[1+\lambda H^{-\frac{1}{2}} B^{\prime} H^{-\frac{1}{2}}\right]^{-1} H^{-\frac{1}{2}} } \\
= & H^{-\frac{1}{2}}\left[\sum_{n=0}^{\infty}\left(-\lambda H^{-\frac{1}{2}} B^{\prime} H^{-\frac{1}{2}}\right)^{n}\right] H^{-\frac{1}{2}} .
\end{aligned}
$$

This is bounded by the sum

$$
\|R(\lambda)\| \leqslant\left(\lambda^{2}+s\right)^{-1} \sum_{n=0}^{\infty}\left(\frac{\lambda\left\|B^{\prime}\right\|}{\lambda^{2}+s}\right)^{n}=\left(\lambda^{2}-\lambda\|B\|+s\right)^{-1} .
$$

This proves that (4.19) converges and $\lambda$ satisfying (4.18) is in $\rho(L)$.

Another result we shall need is that $\rho(L)$ and $\sigma(L)$ are symmetric about the imaginary- $\lambda$ axis, which follows directly from (4.11) and the assumed properties of $B^{\prime}$ and $C^{\prime}$ : if $\lambda$ is in $\rho(L)$ then so is $-\lambda^{*}$. We can now provide restrictions on the spectrum which are illustrated in figure 1 . This is essentially a generalization of Howard's circle theorem (Drazin \& Howard 1966).

THEOREM 1. The spectrum $\sigma(L)$ consists of the union of $(a)$ a subset of the imaginary axis with (b) a subset of those points for which $|\lambda|^{2} \leqslant-\min (0, s)$ and $|\operatorname{Im} \lambda| \leqslant \frac{1}{2}\left\|B^{\prime}\right\|$.

Proof. Suppose, starting at $\lambda_{0}$ in $\rho(L)$, we approach a point $\sigma_{0}$ on the boundary of the spectrum along a curve in $\rho(L)$. As $\lambda \rightarrow \sigma_{0}$, equation (4.17) shows us that 
$\|R(\lambda)\| \rightarrow \infty$. There must therefore exist a sequence of vectors $\boldsymbol{e}_{\lambda}$ of unit norm in $H^{\prime}$ such that the vectors

$$
\boldsymbol{\eta}_{\lambda}=R(\lambda) \boldsymbol{e}_{\lambda}, \quad \lambda \in \rho(L), \quad \lambda \rightarrow \sigma_{0},
$$

have unboundedly increasing norm. Inverting this equation and dividing by $\left\|\boldsymbol{\eta}_{\lambda}\right\|$ gives

$$
L(\lambda) \boldsymbol{\eta}_{\lambda} /\left\|\boldsymbol{\eta}_{\lambda}\right\|=\boldsymbol{e}_{\lambda} /\left\|\boldsymbol{\eta}_{\lambda}\right\| \rightarrow 0 .
$$

That is, there must exist a sequence of normalized vectors $\xi_{\lambda}\left(=\boldsymbol{\eta}_{\lambda} /\left\|\boldsymbol{\eta}_{\lambda}\right\|\right)$ for which

$$
L(\lambda) \xi_{\lambda} \rightarrow 0, \quad \lambda \in \rho(L), \quad \lambda \rightarrow \sigma_{0} .
$$

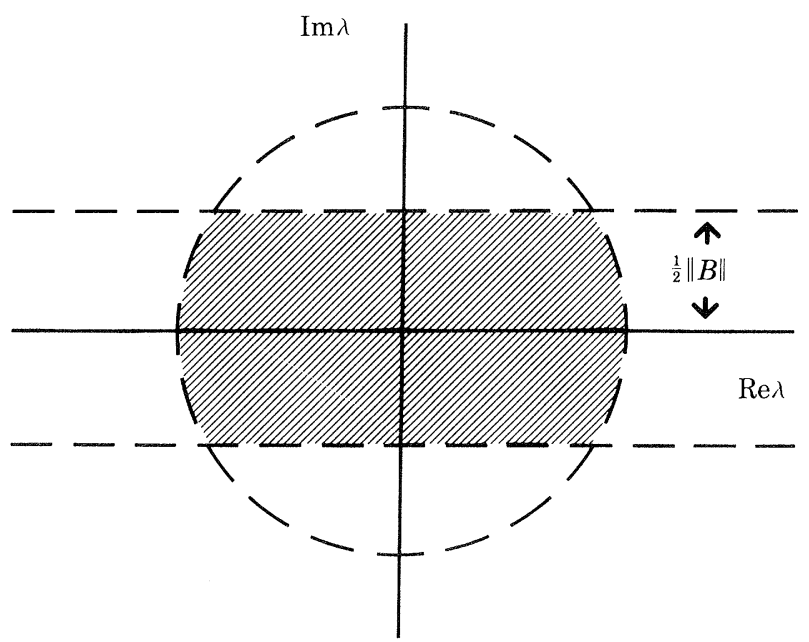

FIGURE 1. Limits on the spectrum of normal modes of a rotating star. The circle has radius $|s|^{\frac{1}{2}}$, where $s$ is the (negative) lower bound of $C^{\prime}$. If $C^{\prime}$ has a non-negative lower bound, the circle vanishes. The spectrum consists of some points on the imaginary axis and some inside the shaded region of the circle. Since unstable modes correspond to points in the right half-plane, if $s$ is non-negative then there are no unstable modes. But if $s$ is negative there is no guarantee of instability: there are only limits on the spectrum.

We shall place bounds on $\sigma(L)$ by determining necessary conditions for this to happen.

It is necessary (but not sufficient) that

or

$$
\left\langle\xi_{\lambda}, L(\lambda) \xi_{\lambda}\right\rangle \rightarrow 0,
$$$$
\lambda^{2}+\mathrm{i} \lambda b(\lambda)+c(\lambda) \rightarrow 0,
$$

where

$$
b(\lambda)=-\mathrm{i}\left(\xi_{\lambda}, B^{\prime} \xi_{\lambda}\right), \quad c(\lambda)=\left(\xi_{\lambda}, C^{\prime} \xi_{\lambda}\right)
$$

are both real. Equation (4.21) has solutions $\lambda_{ \pm}$given (implicitly) by

$$
\lambda \rightarrow \lambda_{ \pm}=-\frac{1}{2} \mathrm{i} b\left(\lambda_{ \pm}\right) \pm \mathrm{i}\left[\frac{1}{4} b^{2}\left(\lambda_{ \pm}\right)+c\left(\lambda_{ \pm}\right)\right]^{\frac{1}{2}} .
$$


The boundaries of $\sigma(L)$ must be solutions of (4.22). The constraints that $b$ and $c$ are real, that $c$ is bounded below by $s$, and that $|b|$ is bounded by $\left\|B^{\prime}\right\|$ lead to the limits given in the theorem.

We shall need two more results for the time-evolution problem. We know now that $R(\lambda)$ is analytic at least everywhere outside the strip $|\operatorname{Re}(\lambda)| \leqslant-s$. Its behaviour at infinity is of interest.

LEMma 1. The resolvent $R(u+\mathrm{i} v)$ (with $u, v$ real) is a bounded function of $v$ as $|v| \rightarrow \infty$ for fixed $u \neq 0$ and of $u$ as $|u| \rightarrow \infty$ for fixed $v$.

Proof. The proof is by contradiction. If $\|R(u+\mathrm{i} v)\|$ is not bounded then equation (4.21) again applies along the given paths. The imaginary and real parts of (4.21) respectively establish the result for the two classes of paths.

Lemma 2. If $\xi$ is in the domain of $C^{\prime}$ then the following asymptotic bounds hold along the paths of lemma 1 at each point in the star:

$$
\begin{gathered}
|R(\lambda) \xi| \approx|\lambda|^{-2}, \\
\left|R(\lambda)\left(\lambda+B^{\prime}\right) \xi\right| \approx|\lambda|^{-1} .
\end{gathered}
$$

Proof. This follows from lemma 1 and a variant of identity (4.16) for vectors in the domain of $C^{\prime}$ :

$$
\lambda^{-1}\left(\lambda^{2}+\lambda B^{\prime}+C^{\prime}\right)^{-1} C^{\prime}=\lambda^{-1}-\left(\lambda^{2}+\lambda B^{\prime}+C^{\prime}\right)^{-1}\left(\lambda+B^{\prime}\right) .
$$

If we operate on a fixed vector $\xi$ with both sides of this equation, lemma 1 gives (4.24). Dividing by $\lambda$ then gives (4.23). It is important to restrict $\xi$ to the domain of $C$; counter-examples can be found for other $\xi$.

\section{(c) Evolution from initial data}

We have studied the eigenvalue problem in order to solve the initial-value problem, equation (4.1). If the solution $\xi(t)$ which evolves from given initial data $\xi_{t}(0)$ and $\xi(0)$ is bounded in time by $\mathrm{e}^{u_{0} t}$, then, following Case (1960), we can find its Laplace transform

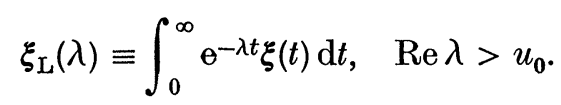

It satisfies the equation

$$
\left(\lambda^{2}+\lambda B^{\prime}+C^{\prime}\right) \xi_{\mathrm{L}}(\lambda)=\left(\lambda+B^{\prime}\right) \xi(0)+\xi_{\mathrm{t}}(0)
$$

or

$$
\xi_{\mathrm{L}}(\lambda)=R(\lambda) \xi_{\mathrm{t}}(0)+R(\lambda)\left(\lambda+B^{\prime}\right) \xi(0) .
$$

The time-development of $\xi$ can be recovered from the usual Laplace-inversion integral

$$
\xi(t)=\frac{1}{2 \pi \mathrm{i}} \int_{-\mathrm{i} \infty+u_{1}}^{\mathrm{i} \infty+u_{1}} \mathrm{e}^{\lambda t} R(\lambda)\left[\xi_{\mathrm{t}}(0)+\left(\lambda+B^{\prime}\right) \xi(0)\right] \mathrm{d} \lambda,
$$


where the integral is over the line $\operatorname{Re}(\lambda)=u_{1}>u_{0}$ in the complex plane. Clearly, the spectral points (singularities of $R(\lambda)$ ) will be crucial.

All of this follows from the assumption that $\xi(t)$ was sufficiently well behaved. We, however, have the converse problem: given $\xi_{t}(0)$ and $\xi(0)$, what properties of $R(\lambda)$ guarantee that (4.27) exists and gives the correct solution to (4.1)? Our answer uses the following theorem in Laplace transform theory (Ditkin \& Prudnikov 1965). The condition

$$
\sup _{u>u_{0}} \int_{-\infty}^{\infty}\left|f_{\mathrm{L}}(u+\mathrm{i} v)\right|^{2} \mathrm{~d} v<\infty
$$

is necessary and sufficient for a function $f_{L}(\lambda)$, analytic in the half-plane $\operatorname{Re}(\lambda)>u_{0}$, to be the Laplace transform of a function $f(t)$ for which

$$
\int_{0}^{\infty}|f(t)|^{2} \mathrm{e}^{-2 u_{0} t} \mathrm{~d} t<\infty
$$

Since we already know that $R(\lambda)$, and therefore $\xi_{\mathrm{L}}(\lambda)$, is analytic for $\operatorname{Re}(\lambda)>|s|^{\frac{1}{2}}$, all we need prove is the following.

Lemma 3. If $\xi(0)$ and $\xi_{t}(0)$ are in the domain of $C^{\prime}$, then for any $\epsilon>0$, and at each point in the star

$$
\sup _{u>|s|^{\left.\right|^{+}}+\epsilon} \int_{0}^{\infty}\left|R(u+\mathrm{i} v)\left[\xi_{\mathrm{t}}(0)+\left((u+\mathrm{i} v)+B^{\prime}\right) \xi(0)\right]\right|^{2} \mathrm{~d} v<\infty .
$$

Proof. This follows directly from lemma 2.

We can therefore state the principal result of this section.

Theorem 2. Equation (4.27) gives the development in time of initial data in the domain of $C^{\prime}$. Every solution is bounded in time, in the integral sense of (4.29), by $\exp \left(\lambda_{0} t\right)$, where $\lambda_{0}$ is any real number larger than the largest real part of any point in the spectrum $\sigma(L)$.

Proof. The first statement has already been proved. The second follows from deforming the path of integration to be as close to the spectrum as possible. It is important for $\lambda_{0}$ to exceed the largest real part of the spectrum, because it may happen that the normal mode associated with a point $\sigma_{0}$ in the spectrum will grow as (polynomial or infinite series in $t$ ) $\times \mathrm{e}^{\sigma_{0} t}$.

Theorem 2 proves a weak kind of completeness useful for stability analysis: if one finds that the spectrum lies entirely on the imaginary axis (no unstable modes) then there are no exponentially growing solutions to the initial-value problem.

Before leaving this subject, we should mention that we are very close to having proved that the operator $T$ in $H_{2}$ generates a semigroup, the 'time-evolution' semigroup (Yosida 1957; Bade 1953). We have been unable to use standard semigroup theory, however, because Bade's condition $\left(B^{\prime}\right)$ or Yosida's theorem in lecture 8 are too strong for our problem. 


\section{NORMAL MODES OF ROTATING STARS}

In this section we will make the following assumptions about the spectrum $\sigma(L)$, which we regard as very reasonable and, in principle, provable for the stellar problem.

(i) For $|\operatorname{Im}(\lambda)|>\frac{1}{2}\left\|B^{\prime}\right\|$, where we have shown the spectrum to be confined to the imaginary axis, it consists of isolated eigenvalues of finite multiplicity plus possible accumulation points.

(ii) All points of the spectrum off the imaginary axis are also isolated, of finite multiplicity, and have no accumulation points on the imaginary axis.

We expect that differentially rotating stars should have a continuous piece of the spectrum confined to the imaginary axis $\dagger$ between the bounds set by $B^{\prime}$. The reader will see that our assumptions are actually much stronger than we need in this section, but we make them for the sake of definiteness. They are illustrated in figure 2 .

A normal mode is a solution associated with a single point of the spectrum. If we deform the contour of integration that defines $\xi(t)$ in the Laplace-inversion integral by pushing the contour through the spectrum as shown in figure 2 , then the result is a sum over a finite number of integrals along the contours $\mathscr{C}_{j}, \mathscr{C}_{+}$, and $\mathscr{C}_{-}$:

$$
\xi(t)=\frac{1}{2 \pi \mathrm{i}} \sum_{j} \oint_{\mathscr{C}_{j}} \mathrm{e}^{\lambda t} \xi_{\mathrm{L}}(\lambda) \mathrm{d} \lambda+\frac{1}{2 \pi \mathrm{i}} \oint_{\mathscr{C}_{+}} \mathrm{e}^{\lambda t} \xi_{\mathrm{L}}(\lambda) \mathrm{d} \lambda+\frac{1}{2 \pi \mathrm{i}} \oint_{\mathscr{C}_{-}} \mathrm{e}^{\lambda t} \xi_{\mathrm{L}}(\lambda) \mathrm{d} \lambda .
$$

Each contour $\mathscr{C}_{j}$ encircles either: (i) an isolated point, (ii) an accumulation point and all the spectral points in some neighbourhood of it, which can be chosen as small as we like, or (iii) the whole of the continuous spectrum and points accumulating to it. The contours $\mathscr{C}_{+}$and $\mathscr{C}_{-}$include the high frequency contributions, and can be made to intersect the axis as far away as we wish. Because the integral (4.26) converges, the contributions from $\mathscr{C}_{ \pm}$can be made arbitrarily small, and is this limiting sense can be dropped from (5.1).

We now want to define the normal mode associated with any spectral point. As we pointed out in the spherical case, a normal mode is defined by its initial data, both $\xi(0)$ and $\xi_{\mathrm{t}}(0)$. It is natural, then, to see them as elements of $H_{2}=H^{\prime} \oplus H^{\prime}$. If we recall the contribution of contour $\mathscr{C}_{j}$ to the Laplace-inversion integral,

$$
\xi_{j}(t)=\frac{1}{2 \pi \mathrm{i}} \oint_{\mathscr{C}_{j}} \mathrm{e}^{\lambda t} R_{\lambda}(L)\left[\left(\lambda+B^{\prime}\right) \xi(0)+\xi_{\mathrm{t}}(0)\right] \mathrm{d} \lambda,
$$

we see that it is nothing more than the first line of the following equation in $H_{2}$ :

$$
\left(\begin{array}{l}
\xi_{j}(t) \\
\eta_{j}(t)
\end{array}\right)=\frac{1}{2 \pi \mathrm{i}} \oint_{\mathscr{C}_{j}} \mathrm{e}^{\lambda t} R_{\lambda}(L)\left(\begin{array}{cc}
\lambda+B^{\prime} & 1 \\
-C^{\prime} & \lambda
\end{array}\right)\left(\begin{array}{l}
\xi(0) \\
\eta(0)
\end{array}\right) \mathrm{d} \lambda
$$

$\dagger$ Friedman \& Schutz (1978 $b$ ) have shown that trivial perturbations contribute a continuous spectrum like this. It would be interesting to know whether the spectrum of non-trivial modes is discrete even in this region! 
or

$$
\zeta_{j}(t)=\frac{1}{2 \pi \mathrm{i}} \oint_{\mathscr{C}_{j}} \mathrm{e}^{\lambda t} R_{\lambda}(T) \zeta(0) \mathrm{d} \lambda .
$$

It is not hard to see that the second line of (5.2) is just the inversion integral for $\mathrm{d} \xi_{j}(t) / \mathrm{d} t$ once the identity (4.15) has been applied. (The integral over the $1 \mathrm{in}$ (4.15) vanishes.)

Consider the case where $\mathscr{C}_{j}$ contains only one point of the spectrum, $\lambda=\sigma_{j}$. Then we find it convenient to regard the operator

$$
N_{j}(t)=\oint_{\mathscr{C}_{j}} \mathrm{e}^{\lambda t} R_{\lambda}(T) \mathrm{d} \lambda
$$

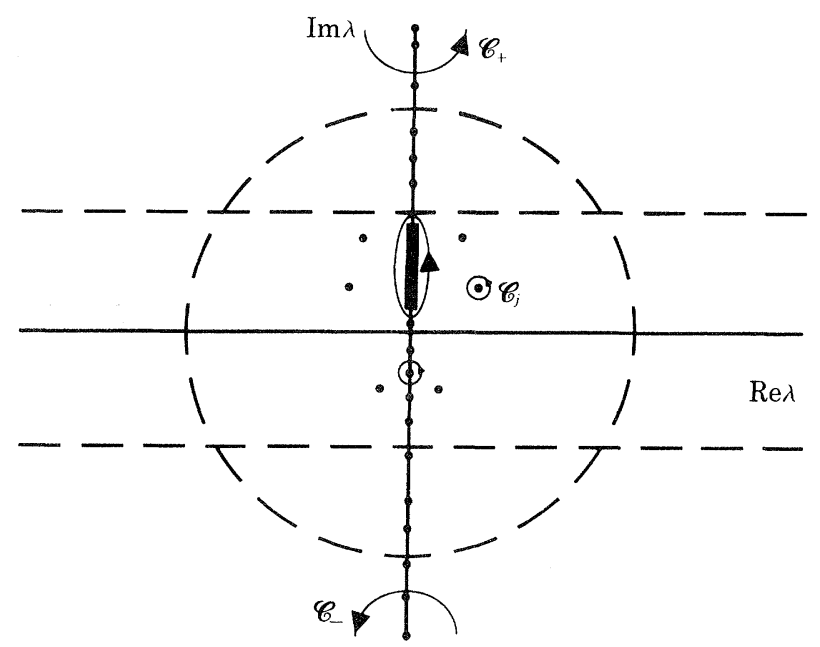

Figure 2. Hypothetical spectrum of normal modes of a rotating star. The heavy line on the imaginary axis is the continuous spectrum. Contours are those used in the Laplaceinv ersion integrals.

itself as the normal mode associated with $\sigma_{j}$. Its value on the initial data $\zeta(0)$ gives the amount of that normal mode in the solution. The operator

$$
P_{j} \equiv N_{j}(0)
$$

is in fact a parallel projection operator (Riesz \& Sz.-Nagy 1955), which projects on to the normal modes at space of $H_{2}$ and commutes with projections for other isolated parts of the spectrum:

$$
P_{j} P_{k}=P_{j} \delta_{j k}
$$

If the spectrum consisted only of isolated points, then the sum (5.1) and the continuity $\xi(t) \rightarrow \xi(0)$ as $t \rightarrow 0$ implies that

$$
\sum_{j} P_{j}=1
$$


which is a statement of the completeness of the normal modes. (Compare (2.5) for selfadjoint operators.)

But the spectrum will be more complicated than this: there will in general be accumulation points and continuous parts. Projections can be defined for accumulation points by a limiting process. Let $\mathscr{C}_{0}$ enclose a sequence of isolated spectral points $\left\{\sigma_{j}, j=1, \ldots\right\}$ with limit point $\sigma_{\infty}$. A projection can be defined for each $\sigma_{j}$, as above, so we define for $\sigma_{\infty}$ the projection

$$
P_{\infty}=\oint_{\mathscr{C}_{0}} R_{\lambda}(T) \mathrm{d} \lambda-\sum_{j=1}^{\infty} P_{j} .
$$

However, when the contour $\mathscr{C}_{0}$ surrounds a continuous part of the spectrum, we know of no method which will define for us a projection operator for each point, or a family of projections as in the selfadjoint case. We are forced to settle for a single projection on to the whole continuous spectrum. If, as we have assumed, this part of the spectrum is confined to the imaginary axis, then it will contribute no instabilities (in the sense of exponentially growing solutions).

What we are able to achieve in the way of completeness for the rotating case is much weaker than for the non-rotating case. The result of most practical value is that the spectrum bounds the growth of all perturbations. The fact that the normal modes are, in some sense, complete is perhaps reassuring for the trustworthiness of the numerical calculations we intend to pursue. In numerical calculations, the problem must be approximated by a finite-dimensional one, whose normal modes are always complete. If this had not been true for the infinite-dimensional case, one would have had to interpret the numerical work cautiously.

What do the normal modes themselves look like? This is another question whose answer is less complete than in the spherical case. For selfadjoint operators, the restriction of the operator to the subspace associated with one of its projection operators reduces the operator to multiplication by the eigenvalue: all vectors in the subspace are eigenvectors. Not so here. It is well known from finite-dimension matrices (cf. Hirsch \& Smale 1974) that the characteristic subspaces of a nonsymmetric operator do not necessarily consist only of eigenvectors; they can contain Jordan chains. In other words, not all operators are diagonalizable. Our projection operators project on to the subspace spanned by the whole chain to the eigenvalue, and these generalized characteristic vectors must be included to obtain completeness. As Bognar (1974) points out, these chains are associated with modes which are not purely exponential in time, but which generally are polynomials in $t$ (whose maximum order is the length of the Jordan chain) times the exponential of the eigenfrequency. Our methods in this paper give no information about these chains except that, if they are infinite in length, the polynomial in time they produce is bounded by any exponential. It would be very useful to have some idea of what order these polynomials may be. At present we have only the example of the Maclaurin spheroids' bar mode (Chandrasekhar 1969), where the symmetry of the problem reduces $H_{2}$ to two dimensions. There the only Jordan chain appears at 
the onset of dynamical instability (i.e. on the imaginary axis), and the corresponding mode has a first-order polynomial in time in it. Is this a general feature, or an accident of the low dimensionality of $H_{2}$ ?

It is a pleasure to acknowledge many helpful discussions with R. Sorkin, D. Evans and D. Harris.

\section{Appendix A. The local buoyancy FRequency}

The purpose of this appendix is to show, in a heuristic way, the relation between the condition we are forced to place on the star in order to guarantee lower-boundedness of $C^{\prime}$ and the local response of the fluid to a perturbation.

Consider the adiabatic displacement of a fluid element from its original position $r$ to a new position $r+\xi$, in such a manner that the element acquires the pressure and angular velocity of the background star at its new position. Its density, $\rho_{\mathrm{e}}$ differs (in general) from that of the background, $\rho_{\mathrm{b}}$, at the new position. The force per unit volume it feels is

$$
\boldsymbol{F}=-\rho_{\mathrm{e}} \nabla \Phi-\nabla p+\rho_{\mathrm{e}} \Omega^{2} \hat{\tilde{\omega}}
$$

where $\hat{\tilde{\omega}}$ is the unit vector in the cylindrical radial direction. The equilibrium equation of the background gives us

and so we conclude

$$
0=-\rho_{\mathrm{b}} \nabla \Phi-\nabla p+\rho_{\mathrm{b}} \Omega^{2} \hat{\tilde{\omega}}
$$

$$
\boldsymbol{F}=\left(\rho_{\mathrm{b}}-\rho_{\mathrm{e}}\right)\left(\nabla \Phi-\Omega^{2} \hat{\tilde{\omega}}\right)=\left(\rho_{\mathrm{e}}-\rho_{\mathrm{b}}\right) \nabla p / \rho_{\mathrm{b}}
$$

Now, to first order in $\xi$ we have

$$
\rho_{\mathrm{e}}=\rho+\Delta_{\xi} \rho=\rho+\frac{\rho}{\gamma p} \Delta_{\xi} p=\rho+\frac{\rho}{\gamma p} \xi \cdot \nabla p
$$

and

$$
\rho_{\mathrm{b}}=\rho+\xi \cdot \nabla \rho,
$$

the first equation following from the adiabatic and isobaric nature of the perturbation ( $\Delta$ denoting the Lagrangian change). So we have

$$
\boldsymbol{F}=\boldsymbol{\nabla} p(\xi \cdot \mathscr{S}),
$$

where $\mathscr{S}$ is the vector Schwarzschild discriminant

$$
\mathscr{S}=(1 / \rho) \nabla \rho-(1 / \gamma p) \nabla p .
$$

The initial acceleration away from its original position in the direction $\xi$ is $(\xi \cdot \nabla p)$ $(\xi \cdot \mathscr{S}) /(\rho|\xi|)$, and this leads to a motion whose square 'resonant frequency' is

$$
N^{2}(\xi)=(\xi \cdot \nabla p) \quad(\xi \cdot \mathscr{S}) /\left(\rho|\xi|^{2}\right) .
$$

When $N^{2}(\xi)>0$ this is a generalization of the (squared) Brunt-Viäsäla frequency (Eckart 1960; Viäsäla 1925). When $N^{2}<0$ we have exponential growth. 
If the star were not rotating then the derivation we have just given would be reasonably watertight. When rotation is present it is merely indicative. In fact, however, our results in $\S 4$ show that if $N^{2}(\xi)$ is bounded then no perturbation grows arbitrarily rapidly. In that sense, this heuristic argument has some plausibility.

\section{Appendix B. Lower bound on a part of $C^{\prime}$}

In this appendix we go beyond Hunter's (1977) calculation to get an explicit form for the lower bound of $C_{3}^{\prime}$, which may have some interest. As Hunter shows, we can write

$$
c_{3}(\xi)\langle\xi, \xi\rangle=\int \rho\left[\left(\xi \cdot \nabla \Omega^{2}\right)\left(\xi_{1} x_{1}+\xi_{2} x_{2}\right)+\Omega^{2}\left(\xi_{1}^{2}+\xi_{2}^{2}\right)\right] \mathrm{d} V .
$$

It will be more convenient to introduce the cylindrical coordinate $\varpi$,

$$
\varpi^{2}=x_{1}^{2}+x_{2}^{2}
$$

and write the integrand of (B 1) as

$$
\rho \xi^{i} \xi^{j}\left[\Omega^{2} P_{i j}+\frac{1}{2} \nabla_{i}\left(\varpi^{2}\right) \nabla_{j}\left(\Omega^{2}\right)\right]
$$

where $P_{i j}$ is the projection operator orthogonal to the $z$ direction. The minimum value of this quadratic form in $\xi$ at any point is simply $\rho|\xi|^{2}$ times the minimum eigenvalue of the matrix in (B 2). It is simple to verify that this eigenvalue, denoted by $\beta$, is

$$
\beta=\min \left\{\left(\varpi \Omega^{2}\right)_{, \varpi}, \frac{1}{2} \Omega^{2}-\frac{1}{2}\left[\Omega^{4}+\left(\left(\varpi \Omega^{2}\right), z\right)^{2}\right]^{\frac{1}{2}}\right\} .
$$

If we extend the definition of $\beta$ to be the minimum of (B 3) over the entire star, then we have proved

$$
c_{3}(\xi) \geqslant \beta
$$

\section{REFERENCES}

Bade, W. G. 1953 Pacific J. Math. 3, 257-290.

Bardeen, J. M. 1975 I.A.U. Symposium 69, Dynamics of Stellar Systems (ed. A. Hayli), p. 297. Dordrecht: Reidel.

Bardeen, J. M., Friedman, J. L., Schutz, B. F. \& Sorkin, R. I977 Astrophys. J. 217, L49-L53.

Barston, E. M. I967-74 J. Math. Phys.: 8, 523 (1967); 8, 1886 (1967); 9, 2069 (1968); 12, $1116(1971) ; 12,1867(1971) ; 13,720(1972) ; 15,675$ (1974).

Bognar, J. I974 Indefinite inner-product spaces. Berlin-Heidelberg-New York: Springer.

Case, K. M. 1960 Phys. Fluids 3, 143-148.

Chandrasekhar, S. 1969 Ellipsoidal figures of equilibrium. New Haven, Connecticut: Yale University Press.

Chandrasekhar, S. \& Lebovitz, N. R. 1968 Astrophys. J. 152, 267.

Comins, N. 1978 Ph.D. thesis, University College, Cardiff, Wales.

Detweiler, S. L. \& Lindblom, L. 1977 Astrophys. J. 211, 565.

Ditkin, V. A. \& Prudnikov, A. P. 1965 Integral transforms and operational calculus. London: Pergamon.

Drazin, P. G. \& Howard, L. N. 1966 Adv. appl. Mech. 9, 1-89.

Eckart, C. 1960 Hydrodynamics of oceans and atmospheres. London: Pergamon. 
Eisenfield, J. I969 J. math. Anal. appl. 26, 357-375.

Fricke, K. \& Kippenhahn, R. I972 A. Rev. Astron. Astrophys. 10, 45.

Friedman, J. L. \& Schutz, B. F. I978a Astrophys. J. 221, 937-957.

Friedman, J. L. \& Schutz, B. F. $1978 b$ Astrophys. J. 222, 281-296.

Hirsch, M. N. \& Smale, S. 1974 Differential equations, dynamical systems, and linear algebra. London: Academic Press.

Hunter, C. 1977 Astrophys. $J .213,497-517$.

Lebovitz, N. R. I965a Astrophys. J. 142, 229.

Lebovitz, N. R. I965b Astrophys. J. 142, 1257.

Lynden-Bell, D. \& Ostriker, J. P. 1967 Mon. Not. R. astr. Soc. 136, 293.

Papaloizou, J. \& Pringle, J. E. 1978 Mon. Not. R. astr. Soc. 182, 423-442.

Riesz, F. \& Sz.-Nagy, B. 1955 Functional analysis. New York: Ungar.

Stewart, J. I975 Astron. Astrophys. 42, 95-101.

Viäsäla, V. 1925 Soc. Sci. Fennica, Commentationes Phys.-Math. II, 19, 37.

Yosida, K. 1957 Lectures on semi-group theory. Bombay: Tata Institute for Fundamental Research. 